\title{
CASE REPORT: ABDOMINAL WALL SEPSIS AND NECROSIS SECONDARY TO TRAUMATIC PERFORATION OF THE AFFERENT LOOP OF A GASTRO-JEJUNOSTOMY
}

\section{MA Galil, MO Adelekan, A Sowinski, Department of Surgery; S Richards, Department of Microbiology; West Cumberland Hospital, Whitehaven}

A 66-year-old man was admitted to the medical ward as an emergency, feeling generally unwell and complaining of severe pain in the upper abdomen for eight days. He gave a history of slipping on ice ten days previously. There were no significant gastrointestinal symptoms prior to his fall.

His past medical history included

- paranoid depression, diagnosed seven years previously

- investigations for haemoptysis three months prior to admission (CT scan showed appearances in keeping with longstanding inactive TB)

- partial gastrectomy for peptic ulcer thirty years ago

- two further abdominal operations for reasons unknown.

On examination he was seriously ill with peripheral cyanosis. Pulse rate was $110 / \mathrm{min}$, blood pressure $116 / 70 \mathrm{mmHg}$. The respiratory rate was $20 / \mathrm{min}$ and his temperature was $36^{\circ} \mathrm{C}$. Abdominal examination revealed vertical and transverse scars in the upper abdomen. There was gross cellulitis in the upper abdomen with a large fluctuant swelling in both hypochondrial regions.

Haematological tests showed a white cell count of $34.2 \mathrm{x}$ $10 / \mathrm{L}$, platelets $7 \times 10 / \mathrm{L}$, sodium of $119 \mathrm{mmol} / \mathrm{L}$, blood urea $31.1 \mathrm{mmol} / \mathrm{L}$ and creatinine $160 \mathrm{umol} / \mathrm{L}$.

A dirty-coloured fluid aspirated from the right hypochondrial region, which showed no pus cells but a large number of Gram-positive cocci and Gram-positive rods. Subsequent cultures of the fluid yielded a very mixed growth of Streptococcus milleri, Staphylococcus aureus, Bacillus licheniformis, Bacillus pumilus, Clostridium sporogenes/ Botulinus (indistinguishable by the reference laboratory).

A working diagnosis was made of abdominal wall sepsis secondary to either necrotising fasciitis or a retroperitoneal perforated viscus. The overall picture was of septicaemia with severe thrombocytopenia.

Following vigorous resuscitation, which included intravenous fluids, intravenous Ciprofloxin, large doses of Benzyl Penicillin and six units of platelet transfusion, the abdominal wall collections were explored in theatre. The left hypochondrial collection contained bile. At this stage the patient's condition became very critical and further surgery was considered unsafe. The wounds were left open and packed with iodine-based antiseptic dressing. The patient was ventilated and transferred to the intensive care unit (ICU) post-operatively.
His general condition deteriorated due to progressive septicaemia and it was decided that explorative laparotomy was his only chance of survival. The findings were as follows:

- necrotic anterior abdominal wall, approximately a litre of pus mainly in the lower abdomen and multiple adhesions from the previous surgery which made access very difficult

- the afferent loop of the gastro-jejunostomy including the third part of the duodenum had necrosed and perforated

- the necrotic bowel was resected, a cholecysto-jejunostomy was performed using the afferent loop of the gastrojejunostomy, and a jejuno-jejunostomy distal to the cholecysto-jejunostomy was performed

- a feeding jejunostomy tube was inserted and a silastic tube drain was positioned near the duodenojejunal flexure to create a controlled biliary fistula

He was returned to ICU where he died an hour later.

\section{DISCUSSION}

This patient had a Billroth II partial gastrectomy for duodenal ulcer thirty years previously. Laparotomy for septicaemia revealed extensive adhesions. Some were band-shaped, forming potential gates for internal herniation. It is likely that the afferent loop had herniated and ruptured when the patient slipped on ice ten days before presentation, and subsequent infection involved the abdominal wall, with sepsis and necrosis.

Necrotising fasciitis of the chest wall secondary to gastric perforation has been reported following Nissen Fundoplication $^{(1)}$ but the onset occurred in the immediate post-operative period.

A search of the literature has not revealed a similar case.

\section{REFERENCE}

1 Viste A, Vindenes H, Gjerde S. Herniation of the stomach and necrotising chest wall infection following laparoscopic Nissen fundoplication. Surgical Endoscopy 1997;11:10291031 